\title{
Network Selection and Resource Allocation Games for Wireless Access Networks
}

\author{
Ilaria Malanchini, Member, IEEE, Matteo Cesana, Member, IEEE, and Nicola Gatti
}

\begin{abstract}
Wireless access networks are often characterized by the interaction of different end users, communication technologies, and network operators. This paper analyzes the dynamics among these "actors" by focusing on the processes of wireless network selection, where end users may choose among multiple available access networks to get connectivity, and resource allocation, where network operators may set their radio resources to provide connectivity. The interaction among end users is modeled as a noncooperative congestion game, where players (end users) selfishly select the access network that minimizes their perceived selection cost. A method based on mathematical programming is proposed to find Nash equilibria and characterize their optimality under three cost functions, which are representative of different technological scenarios. System level simulations are then used to evaluate the actual throughput and fairness of the equilibrium points. The interaction among end users and network operators is then assessed through a two-stage multileader/multifollower game, where network operators (leaders) play in the first stage by properly setting the radio resources to maximize their users, and end users (followers) play in the second stage the aforementioned network selection game. The existence of exact and approximated subgame perfect Nash equilibria of the two-stage game is thoroughly assessed and numerical results are provided on the "quality" of such equilibria.
\end{abstract}

Index Terms-Wireless access networks, network selection, congestion games, price-of-stability, price-of-anarchy

\section{INTRODUCTION}

$\mathrm{T}$ HE impressive growth of standards and technologies for wireless communications has dramatically increased the opportunities for mobile users to connect anytime anywhere. End-user equipment often comes with multiple radio interfaces featuring different communication standards, from short range (Zigbee, Bluetooth) to medium/ long range ones (WiFi, WiMAX, 2G, LTE). Moreover, a given geographical area may be "covered" by multiple access network/technologies with different characteristics (bandwidth, access cost), even potentially run by different operators. As an example, 2G/3G cellular systems owned by big Tel-Co operators may provide geographical coverage side by side with multidomain Wireless Local Area Networks (WLANs), and Wireless Mesh Networks (WMN) run by medium/small Wireless Internet Service Providers (WISPs).

Different from the past when wireless connectivity was monopolized by a single operator/technology, the overprovisioning of access networks nowadays allows mobile users to choose among multiple access opportunities on the base of availability, cost and/or quality, eventually achieving a seamless, ubiquitous, and pervasive connectivity experience. To reach this ambitious goal, however, many technical challenges still have to be addressed in different fields. On the network side, signaling architectures are required to effectively support user's mobility among multiple networks and to manage the radio resource

- The authors are with the Dipartimento di Elettronica e Informazione, Politecnico di Milano, Piazza L. da Vinci, 32, 20133 Milan, Italy.

E-mail: ilaria.malanchini@mail.polimi.it, \{cesana,ngatti\}@elet.polimi.it.

Manuscript received 18 Jan. 2012; revised 5 July 2012; accepted 22 Sept. 2012; published online 2 Oct. 2012.

For information on obtaining reprints of this article, please send e-mail to: tmc@computer.org, and reference IEEECS Log Number TMC-2012-01-0028. Digital Object Identifier no. 10.1109/TMC.2012.207. allocation process; on the user's side, effective techniques are required to discover and classify the multiple connectivity opportunities based on quality parameters (actual throughput, access delay, wireless interference level) to steer eventually the dynamic selection of the best connectivity opportunity.

The basic problem of network selection refers to the dynamic and automatic choice of the "best" wireless access network to connect to. In classical cellular systems, the network selection is mainly guided by physical layer parameters, and the mobile terminal often associates to the "best received" base station, i.e., the closest (in terms of received signal strength) to the end-user equipment. Such selection policy is obviously not suited for other wireless access scenarios eventually featuring heterogeneous wireless access technologies. For instance, a WiFi user may favor connecting to a less loaded access point (AP) at larger distance, rather than to a very close, highly loaded one. The specific parameters to drive the selection strategy itself are highly dependent on the specific wireless access technology, thus novel parameters must be accounted in the selection procedure [1]. On the other hand, network operators have to tackle a resource allocation problem that requires the proper setting/planning of the available radio resources (e.g., frequencies, time slots, spreading codes, etc.) throughout their deployed access network infrastructures. The resource allocation driving criteria may include the maximization of the overall revenues for the operator, the maximization of the provided geographical coverage, and/or the maximization of the network spectral efficiency, under tight/loose constraints on the quality perceived by the accessing users.

In this paper, we resort to noncooperative game theoretic tools [2] to analyze the dynamics among users and 
operators by focusing on the processes of wireless network selection, where end users may choose among multiple available access networks to get connectivity, and resource allocation, where network operators may set their radio resources to provide connectivity.

The problem of wireless access network selection is cast as a congestion game [3], where each user is selfish, rational, and selects the access network that maximizes her perceived quality of service. Even if the proposed model is rather general and not dependent on the specific quality measure, we introduce three approximate but consistent measures for the perceived quality of the access process which model realistic technological scenarios. More precisely, one function depends only on the users' perceived interference, whereas the other two also account for the nominal achievable rate upon accessing the network. In this paper, we do not consider connection fees in the game models. This is consistent with network scenarios where, for example, free WiFi access is available in urban areas provided by multiple sources (e.g., FON APs) and/or different operators offer the same service at the same price. The extension to include connection fees is discussed throughout the paper.

Under the assumption, customary in the literature [4], that best response dynamics is much faster than users' mobility dynamics, we can study any setting as a sequence of games in which users are stationary. We prove that with all the three cost functions (CF), we propose there is always at least one pure Nash equilibrium (NE), further providing practical solution algorithm to derive such equilibria which leverages the mathematical programming formulation of the network selection problem. The quality of the equilibria is then characterized in terms of their associated price-ofstability (PoS, see [5]) and price-of-anarchy (PoA, see [6]), defined as the ratio between the best and worst equilibrium (WE), respectively, and the optimal solution, i.e., the one minimizing the social cost without equilibrium constraints. The actual throughput and fairness degree of the equilibrium situations are also assessed through system level simulation using NS2 network simulator [7].

The problem of resource allocation is cast as a multileader/multifollower two-stage game where in the first stage the network operators (i.e., the leaders) play by choosing their resource allocation strategies, while in the second stage the users (i.e., the followers) play the aforementioned network selection game. The aim of the operators is to capture the largest number of accessing users. We formally prove that when the quality measure adopted by the users only depends on the interference level, the twostage game always admits a pure strategy Subgame Perfect Nash Equilibrium (SPE), whereas under the other two quality measures it may not exist. In these latter cases, we leverage the concept of $\epsilon$-SPE to find suboptimal equilibrium situations.

The paper is organized as follows: in Section 2, we set the background for our work by reviewing the literature in the field of network selection. Section 3 defines the network selection game model, while Section 4 provides the mathematical programming formulation to compute the NE. The numerical results on the evaluation of the NE are reported in Section 5. Section 6 finally describes the game model including the network operators. Concluding remarks and comments on ongoing related activities are reported in Section $7 .^{1}$

\section{BACKGROUND}

Work related to the problem of network selection mainly deals with two major aspects: the definition of novel metrics aimed at measuring the perceived quality of accessing users and steering the selection decisions, and the design of communication protocols customized to the multinetwork scenario. The works in [9], [10], and [11] fall in the former research track, and examine quality functions based on different parameters, such as transfer completion time, download throughput, traffic load, and received signal strength, to propose an intelligent strategy for network selection in multiaccess network scenario. A mathematical approach, based on the combination of gray Relational Analysis (GRA) and Analytic Hierarchy Processing (AHP), is adopted in [12] and in [13]. The authors tackle the problem of network selection developing quality functions to determine the user's utility related to different selection choices. A similar mathematical technique is used in [14]. The authors formulate the network selection problem as a multiattribute decision making (MADM) problem that deals with the evaluation of different networks, taking into account many attributes, such as access technology, supported services, and cost.

Research of the latter track usually focuses on specific network scenarios/technologies. Bernaschi et al. [15] propose a vertical handover protocol to handle the user mobility between WLAN and cellular systems. The problem of load balancing in 802.11 WLAN is studied in [16] and [17]. The former proposes an intelligent association control to obtain the fairest solution, in terms of max-min fairness, whereas the latter proposes a mechanism to drive mobile users toward the most appropriate point of access to the network, taking into account both user preferences and network context.

Referring to IEEE 802.11-based networks, Lee and Miller address in [18] the problem of selecting among several 802.11-based APs, by proposing an effective solution to distribute roaming information to the users, which can be used to discriminate in the access phase. The authors of [19] and [20] propose decentralized approaches to choose among multiple APs aiming at achieving an efficient and fair share of wireless-access resources. In [21], the authors describe a methodology for evaluating the potential bandwidth between a client and an AP based on delays experienced by beacon frames. The potential bandwidth is used as the metric adopted by users in the association phase.

In this scenario, even if game theory provides effective tools to evaluate the dynamics involved in the network selection procedures, few works have appeared so far on this specific topic. Interesting overviews on the potentials of game theory in the field of network selection are provided

1. Please refer to the supplemental appendix [8], which can be found on the Computer Society Digital Library at http://doi.ieeecomputersociety.org/ 10.1109/TMC.2012.207, for the formal proofs of Propositions 6.1, 6.2, 6.3, and additional numerical results. 
in [22] and [23]. Mittal et al. [24] resort to a noncooperative game to model the association process to WiFi-based APs. The cost function each user aims at minimizing depends on the access point load and on the distance the associating device needs to travel to being actually able to associate with the desired access point.

WiFi association is addressed through game theoretic tools also in [25], [26], and [27]. All the three works consider noncooperative game models with the users trying to selfishly minimize a cost function that depends on the current congestion of the WiFi access points. In [25] and [26], the cost function depends only on the current congestion level of the access points, whereas Chen [27] introduces a cost function that includes also the association fee each user has to pay to get access from a specific access point. Besides analytical modeling of the network selection game, $\mathrm{Xu}$ et al. [26] also introduce a practical association protocol for Wireless LANs; namely, the authors propose to let APs broadcast information on the current congestion level such that the accessing user can dynamically play their best reply strategies which are proven to converge to an equilibrium. In [28], the authors model the interaction of users and providers as a two-stage multi-leader-multifollower game. In particular, providers compete on the prices and users choose the demand to maximize their payoff. A common feature of the aforementioned works is that they all consider the case of atomic players, with each single player contributing to the costs/utilities of the others in nonnegligible manner.

On the other hand, nonatomic games are considered in [29], [30] and [31]. Namely, Shakkottai et al. [29] consider the case of a single device that can split its traffic across multiple access points it is associated with. Different from the present work, the game model used in their work comes from the family of population games, which are nonatomic, that is, the contribution to the cost of each user is assumed to be negligible. A similar population game model is analyzed in [30] in case the actual throughput perceived by the accessing user depends on transmission/scheduling policies of the network operator. Nonatomic games are also used in [31] to model the problem of selecting the best network. The authors study the loss of efficiency of the user equilibrium. In these games, the proposed cost function depends on the content download delay.

Game theory has been widely used to address also resource allocation problems. Niyato and Hossain propose in [32] a game-theoretic approach for studying bandwidth allocation in heterogeneous wireless networks. Different from our work, the focus is on resource allocation only, and the problem is cast as a bankruptcy (cooperative) game, where different networks form a coalition to provide bandwidth to the end users. The concepts of core and Shapely value are used to determine the quality of the bandwidth allocation. A cooperative game is used in [33] to model the allocation of bandwidth within the several access technologies further managing the distribution of excess bandwidth among operators.

Noncooperative games are used in the field of resource allocation in [32], [34], [35] and [36]. In [34], the focus is on the problem of bandwidth allocation in 802.16-like networks, whereas, Niyato and Hossain [32] introduce a noncooperative game to model the interactions of different access networks (WLAN, cellular systems, and WMAN). In this work, the authors derive both long-term and short-term criteria to allocate bandwidth within different technologies to incoming users. A similar noncooperative scenario in the field of resource allocation is addressed in [36], which addresses the competition of WLANs sharing unlicensed frequency bands. A stage-based noncooperative game is proposed to analyze competition scenarios between two wireless networks.

The aforementioned manuscripts either assume cooperation among network operators, or only focus on the resource allocation problem. Differently, in this paper, we provide a comprehensive framework that models the problem of network selection, investigating different strategies (cost/utility functions) that may fit different access technologies. We address the problem by resorting to congestion games, which provide a powerful tool to represent situations where resources are shared among/ congested by multiple players. Moreover, differently than the reference literature, we extend the network selection model to include operators in the competitive dynamics. Preliminary work along these lines appears in [37] and [38].

\section{The Network Selection Game}

\subsection{The Reference Scenario}

We consider a reference scenario composed of $m$ access points ${ }^{2}$ and $n$ users, where each AP is tuned on a specific radio resource and each user can choose the AP to connect to. We denote an AP by $a$ and the set of APs by $A$; we denote a radio resource available to AP by $f$ and the set of radio resources by $F$; we denote a user by $u$ and the set of users by $U$. Without loss of generality, we assume that the available radio resources $f$ at each AP $a$ is one from a set $F$ of frequency channels. Each AP $a$ uses a frequency $f$ and is characterized by a coverage area that depends on the transmission range and propagation model. Frequency reuse is allowed among different access points. The network topology defines the APs' number, positions, frequencies, and coverage areas.

\subsection{The Game Theoretic Model}

We model this scenario as a noncooperative game in which users are players and their action is the selection of an AP among the available ones. The availability of an action for a user is determined by the network topology and the user's position. More precisely, each user can select one AP among all the ones whose coverage area includes the user's position. In the model, the coverage areas are arbitrary, while in the experimental setting discussed in Section 5 we will adopt a specific propagation model. We denote by $A_{u}^{f} \subseteq A$ the set of APs transmitting on frequency $f$ and available to user $u$. We report an example of network in Fig. 1, where $m=2, n=10$. Black circles denote users, lines

2. Hereafter, we will use the terms "access points," "access networks," and "networks" interchangeably. In practice, access points can be IEEE 802.11 hot spots, $3 \mathrm{G} / 4 \mathrm{G}$ base stations. 


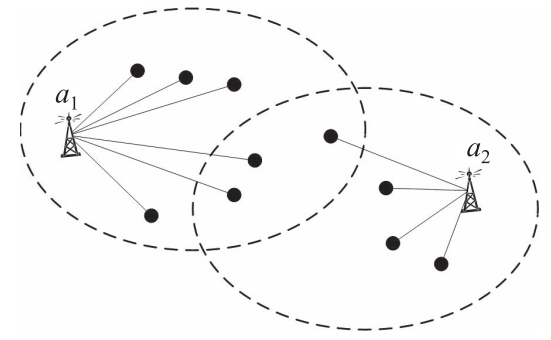

Fig. 1. A network with two access points.

between users and access points denote associations, and dashed lines delimit coverage areas.

Two users selecting different APs that operate on the same frequency will interfere if they are in the range of both APs. Each user $u$ perceives a cost $c_{u}\left(f, z_{u}^{f}\right)$ that depends on the congestion level $z_{u}^{f}$ perceived by user $u$ on frequency $f$ (three different expressions of $c_{u}\left(f, z_{u}^{f}\right)$ will be proposed in the next section). We denote by $X_{u}^{f} \subseteq U$ the set of users that use frequency $f$ and interfere with user $u$. In general, each user $u$ can congest frequency $f$ with a specific weight $\omega_{u}^{f}$. For instance, the congestion weight of a user can be a function of the distance between the user and the AP or of the user's traffic. The congestion level $z_{u}^{f}$ perceived by user $u$ on frequency $f$ is defined as $z_{u}^{f}=\sum_{u \in X_{u}^{f}} \omega_{u}^{f}$. Reasonably, we assume that the cost increases as the congestion level increases, thus $c_{u}\left(f, z_{u}^{f}\right)$ is strictly monotonically increasing in $z_{u}^{f}$.

The model drastically simplifies when all the users congest the APs (and consequently the frequencies) with the same weight $\omega_{u}^{f}=1$. We denote by $x_{u}^{f}$ the cardinality of $X_{u}^{f}$, formally, $x_{u}^{f}=\left|X_{u}^{f}\right|$, i.e., the number of users that connect to frequency $f$ and interfere with user $u$. When all the users congest the APs with the same weight, we have $z_{u}^{f}=x_{u}^{f}$ (the corresponding game is nonweighted).

Each user is rational and behaves selfishly trying to minimize her cost. The solution concept we refer to is the well-known (pure strategy) Nash equilibrium, i.e., an action profile $S^{*}=\left(s_{u_{1}}, \ldots, s_{u_{n}}\right)$, where $s_{u}$ is the action of user $u$, such that no user can reduce her perceived cost by deviating unilaterally from $S^{*}$ [2]. Given an action profile $S$, we denote by $n_{a}$ the number of users that connect to AP $a$.

We consider a congestion game [3] that is: asymmetric (different users can have different available actions), singlechoice (each user can select only one AP), and with playerspecific cost functions (each user can have a different cost function). It is worth noting that different users using the same frequency may perceive different congestions. Furthermore, the game defined above can be reduced to a crowding game [39], i.e., a symmetric single-choice congestion game with player-specific cost functions that are monotonically increasing in the level of congestion. The formal proof of the reduction is presented in [40] for a similar game model. This equivalence leads to a prominent property when the game is nonweighted: it is proved to always admit a pure strategy NE [39]. The literature on congestion games shows that best-response dynamics surely converges to pure strategy NEs, in case NEs do exist [3]. This allows us to study safely only situations where the users' actions are in equilibrium. Therefore, independently
TABLE 1

Summary of the Cost Functions that Are Discussed in the Paper

\begin{tabular}{|c|c|c|c|}
\hline Cost Function & $\omega_{u}^{f}$ & $z_{u}^{f}$ & $c_{u}\left(f, z_{u}^{f}\right)$ \\
\hline CF 1 & 1 & $x_{u}^{f}$ & $x_{u}^{f}$ \\
\hline CF 2 & $T_{u}^{f}$ & $\sum_{u^{\prime} \in X_{u}^{f}} T_{u^{\prime}}^{f}$ & $T_{u}^{f} \cdot z_{u}^{f}$ \\
\hline CF 3 & 1 & $x_{u}^{f}$ & $T_{u}^{f} \cdot x_{u}^{f}$ \\
\hline
\end{tabular}

of the specific definition of $c_{u}\left(f, z_{u}^{f}\right)$, we can focus on algorithms to find pure NEs. When instead the game is weighted, the existence of pure NEs depends on the definition of $c_{u}\left(f, z_{u}^{f}\right)$. We discuss details in the section where we study the weighted congestion game.

\subsection{Cost Functions}

We define three different cost functions, which approximate the actual access cost under different wireless access technologies. We summarize the cost functions in Table 1. It is worth noting that, all throughout the paper, we will refer to "nominal rate/bandwidth" as the rate assigned by the network to the users (in 802.11 this is known as rate adaptation mechanism [41] and usually depends on the received signal strength). In contrast, we refer to "actual throughput" as the actual rate perceived by the user that is usually affected by the surrounding interference and network conditions.

\subsubsection{Cost Function 1: Interference Based}

In general wireless access networks, the quality of service obtained by each user strictly depends on the perceived actual throughput. Since the nominal bandwidth is shared among all connected users, the quality/cost perceived by an accessing user depends on the number of competing users sharing the very same resource. It is, thus, reasonable to introduce a cost function that depends directly on the cardinality of the interfering users. This may well represent the case of access networks characterized by "soft" capacity degradation like the uplink of CDMA-based systems under open loop power control, where the perceived quality of a transmission depends almost exclusively on the interferers number and each transmission/user congests the shared resource evenly [42]. In this case, for all $u \in U, f \in F$, we have

$$
\omega_{u}^{f}=1, \quad z_{u}^{f}=x_{u}^{f}, \quad c_{u}\left(f, z_{u}^{f}\right)=x_{u}^{f} .
$$

The game is nonweighted. In particular, when frequency reuse is not allowed, the game admits an exact potential function. In this case, all the users perceive the same congestion from $f$ and the potential function is the one provided by Rosenthal in [3], formally, $\Psi(S)=\sum_{a=1}^{m} \sum_{k=1}^{n_{a}} k$, where $S$ is the users' strategy profile. (We recall that in a potential game every action profile that minimizes the potential function is a NE.)

\subsubsection{Cost Function 2: Weighted Interference-Rate}

Wireless technologies used in access networks may feature rate-adaptation mechanisms that dynamically adapt the nominal rate to the received signal strength. Therefore, it is worth considering a cost function that accounts both for the number of interferers and for the nominal rate. We 
denote by $R_{u}^{a}$ the rate perceived by user $u$ connecting to $a$. We denote by $T_{u}^{a}$ the inverse of the rate $R_{u}^{a}$ perceived by user $u$ to connect to $a$, i.e., $T_{u}^{a}=\frac{1}{R^{a}}$. Moreover, since the cost function is defined with respect to the chosen frequency, we define

$$
T_{u}^{f}=\min _{a \in A_{u}^{f}} T_{u}^{a},
$$

where $A_{u}^{f}$ is the set of APs available to $u$ using $f$.

The accessing users may then congest the shared resource in different ways. As an example, in IEEE 802.11 access networks, the highest interference is caused by users with the lowest rate due to the well-known performance "anomaly" [43]. Thus, it is reasonable to assume that each user congests the resources with a specific weight that depends on the inverse of the rate the user perceives. Formally, defining $\omega_{u}^{f}$ as the inverse of the rate, we have

$$
\omega_{u}^{f}=T_{u}^{f}, z_{u}^{f}=\sum_{u^{\prime} \in X_{u}^{f}} T_{u^{\prime}}^{f}, c_{u}\left(f, z_{u}^{f}\right)=T_{u}^{f} \cdot z_{u}^{f} .
$$

Note the game is weighted and it does not admit any potential function, and therefore, the results discussed at the end of Section 3.2 cannot be applied, the results discussed in [44] show that when the cost functions are separable, i.e., when the cost is defined as the product of a player-specific parameter and the congestion level, the game always admits a pure NE.

\subsubsection{Cost Function 3: Interference-Rate}

We observe here that the practical implementation of the cost function defined in Section 3.3.2 requires the knowledge for a user of the rate values adopted by all the other users in the network, which may be not feasible or too expensive to achieve. We propose here an approximate cost function that combines both interference and rate, but requires more limited information to be distributed to the users. In case the nominal rate and the number of competing users are available, we may define a cost function that captures the portion of bandwidth achievable to each user, as the current nominal rate divided by the number of interferers.

As done for the previous cost functions, we use the inverse of the rate and we obtain the product between the number of interferers and the inverse of the rate perceived by the considered user. Formally, for all $u \in U, f \in F$, we have

$$
\omega_{u}^{f}=1, \quad z_{u}^{f}=x_{u}^{f}, \quad c_{u}\left(f, z_{u}^{f}\right)=T_{u}^{f} \cdot x_{u}^{f} .
$$

Since in this case the game is nonweighted, the existence of at least one pure strategy NE is guaranteed, as mentioned at the end of Section 3.2. However, the game does not admit any potential function.

\section{FINDING NASH EqUILIBRIA}

The literature on congestion games generally resorts to the minimization of potential functions to calculate Nash equilibria. However, our games do not always admit any potential function. Thus, we propose a solution approach based on a mathematical programming formulation of the network selection problem that can be used in realistic scenario for all the cost functions.
To this end, we introduce the following parameters, defined as $\forall u \in U, f \in F, a \in A$ :

$$
\begin{aligned}
b_{u a} & = \begin{cases}1, & \text { if user } u \text { can select access point } a, \\
0, & \text { otherwise, }\end{cases} \\
d_{u f} & = \begin{cases}1, & \text { if user } u \text { can select frequency } f, \\
0, & \text { otherwise, }\end{cases} \\
t_{a f} & = \begin{cases}1, & \text { if } a \text { transmits on frequency } f, \\
0, & \text { otherwise, }\end{cases} \\
i_{u v f} & = \begin{cases}1, & \text { if users } u \text { and } v \text { potentially } \\
0, & \text { interfere on frequency } f,\end{cases}
\end{aligned}
$$

Given a generic topology, $b_{u a}$ is equal to 1 if user $u$ is within the coverage area of $a$. The value of each $b_{u a}$ (and also the reverse of the nominal rates $T_{u}^{a} \mathrm{~s}$ ) are computed once the propagation model is chosen. Furthermore, $d_{u f}$ is equal to 1 if user $u$ is covered by at least one AP that is using frequency $f$. Then, we define $F_{u}$ as the set of frequencies that user $u$ can choose, i.e., such that $d_{u f}=1$.

We define the assignment of a user to a frequency by introducing a binary decision variable, $\forall u \in U, f \in F$ :

$$
y_{u f}= \begin{cases}1, & \text { if user } u \text { chooses frequency } f \\ 0, & \text { otherwise }\end{cases}
$$

and similarly, we define a binary decision variable for the association of each user to an $\mathrm{AP}, \forall u \in U, a \in A$ :

$$
s_{u a}= \begin{cases}1, & \text { if user } u \text { chooses } \operatorname{AP} a, \\ 0, & \text { otherwise. }\end{cases}
$$

Note that the variable $x_{u}^{f}$ introduced in Section 3 is

$$
x_{u}^{f}=\sum_{v \in U} y_{v f} i_{u v f} .
$$

Recalling that $\omega_{u}^{f}$ is the congestion weight of user $u$ to frequency $f$, the congestion level $z_{u}^{f}$ of frequency $f$ is

$$
z_{u}^{f}=\sum_{v \in U} \omega_{v}^{f} y_{v f} i_{u v f}
$$

With the social cost as the objective function, i.e.,

$$
\sum_{u \in U} \sum_{f \in F} y_{u f} c_{u}\left(f, z_{u}^{f}\right),
$$

the socially optimal network selection is a solution of the following mathematical programming problem:

$$
\min \sum_{u \in U} \sum_{f \in F} y_{u f} c_{u}\left(f, z_{u}^{f}\right)
$$

s.t.

$$
\begin{gathered}
\sum_{a \in A_{u}} s_{u a}=1 \quad \forall u \in U, \\
y_{u f}=\sum_{a \in A_{u}} s_{u a} t_{a f} \quad \forall u \in U, f \in F,
\end{gathered}
$$

where constraints (3) ensure that each user chooses only one AP among available ones, while (4) guarantees that the 
frequency assigned to user $u$ is the frequency $f$ used by AP $a$ if and only if $s_{u a}$ is equal to one.

A (pure strategy) NE can be found by solving the following feasibility problem:

constraints $(3),(4)$

$$
d_{u k} y_{u f} c_{u}\left(f, z_{u}^{f}\right) \leq c_{u}\left(k, z_{u}^{k}+\omega_{u}^{k}\right) \quad \begin{aligned}
& \forall u \in U, f, \\
& k \neq f \in F,
\end{aligned}
$$

where constraints (5) force each user $u$ to select the access point which minimizes $u$ 's cost function, that is, they ensure that if a user unilaterally changes her action, then she cannot reduce her cost (i.e., definition of NE).

The selection of a specific NE can be easily addressed by introducing an objective function. For instance, the worst NE (needed for calculating PoA) can be obtained by maximizing (1), whereas the best NE (needed for calculating PoS) can be obtained by using (2).

For the sake of readability, we present the model using nonlinear constraints. A mixed-integer linear formulation for all the cost functions can be easily obtained.

Finally, it is worth noting that the presented model can be used for all the presented cost functions. Namely, it is used to characterize the optimal allocation and the best/ worst equilibrium of the games.

\section{EXPERIMENTAL Evaluation}

We report an experimental evaluation of the access strategies associated with the three cost functions both in terms of equilibrium inefficiency (i.e., PoA and PoS) and average perceived throughput by the users. We denote by 1,2 , and 3 the cost functions presented in Sections 3.3.1, 3.3.2, and 3.3.3, respectively, as mentioned in Table 1. Furthermore, we consider also the case in which users select the nearest $\mathrm{AP}$, i.e., $c_{u}\left(f, z_{u}^{f}\right)=T_{u}^{f}$, regardless of the number of interferers. We denote this cost function by "nearest-AP." Indeed, according to the propagation model that we have assumed, this case represents the case in which access points are selected on the basis of the received signal strength only. Note that this is not a game anymore (and then it is considered in the throughput evaluation only), but it is relevant because it is the common association policy in 802.11 networks.

\subsection{Experimental Setting}

We consider a multiaccess network deployed on a square area with edge $L$ and composed by $n$ users and $m$ APs with a coverage range of $r$ meters. We have implemented a generator able to create synthetic instances representing the simulated network scenarios. The generating tool randomly draws the position of the $m$ APs and the $n$ users, so that each user is covered by at least one AP. Moreover, in this section, we assume that each AP uses a different frequency, which is the "best" situation for the users because inter-AP interference is null. We relax this assumption in the following section.

For the sake of simulation, we refer to the standard $802.11 \mathrm{~g}$ and we adopt the free space propagation model in [45]:
TABLE 2

Achievable Rates in a 802.11g Scenario

\begin{tabular}{|c|c|c|c|}
\hline $\begin{array}{c}\text { Distance } \\
{[\text { meters }]}\end{array}$ & $\begin{array}{c}\text { SNR } \\
{[\mathrm{dB}]}\end{array}$ & $\begin{array}{c}\text { Rate } \\
{[\text { Mbit } / \mathrm{s}]}\end{array}$ & $\mathrm{T}$ \\
\hline$\leq 10$ & $\geq 25.05$ & 54 & 1.8 \\
\hline$\leq 20$ & $\geq 19.03$ & 48 & 2 \\
\hline$\leq 30$ & $\geq 15.51$ & 36 & 2.7 \\
\hline$\leq 45$ & $\geq 11.99$ & 24 & 4 \\
\hline$\leq 60$ & $\geq 9.49$ & 18 & 5.5 \\
\hline$\leq 75$ & $\geq 7.55$ & 12 & 8.3 \\
\hline$\leq 90$ & $\geq 5.97$ & 9 & 11.1 \\
\hline$\leq 100$ & $\geq 5.05$ & 6 & 16.6 \\
\hline
\end{tabular}

$$
\mathrm{SNR}=10 \log \frac{P_{r}}{N}, \quad P_{r}=P_{t} \cdot G_{t} \cdot G_{r}\left(\frac{\Lambda}{4 \pi d}\right)^{2},
$$

where $P_{t}=0.01 \mathrm{~W}$ is the transmission power, $G_{t}=G_{r}=1$ are the antenna gains, $\Lambda$ is the wavelength and can be derived by frequency that is set to $2.437 \mathrm{GHz}$ and noise power is $N=3 \cdot 10^{-11} \mathrm{~W}$.

To have correct reception, the SNR threshold is set to $5.05 \mathrm{~dB}$ that corresponds to a circular coverage area of radius $r=100$ meters. According to the distance $d$ between the user and the AP, when this is shorter than the radius, the software assigns the largest nominal rate that the user can obtain from that AP (rate adaptation scheme). Table 2 reports, for each distance and SNR, the nominal rate and the corresponding value of $T$ used in our simulations. We assume that $T_{u}^{f}=T, \forall u \in U, f \in F$.

The parameter $T$ used in the model is a normalization of the inverse of rate $R$, so that the number of interferers and the inverse of rate are comparable. In this case, we assume that $T=10^{8} / R$. This normalization could not be effective if the number of users or APs increases. Extensive simulations have shown that an effective normalization should depend on $n$ and $m$ so that the worst case of rate (that corresponds to the maximum value of $T$ ) is comparable with the average number of users per AP. For this reason, we propose the normalization $T_{\max }=(n / m) \beta$, where $T_{\max }$ is the maximum value of $T$ and $\beta$ should be chosen in the interval $[1,4]$ (in our simulations $\beta=3.32$ ).

\subsection{Evaluating Equilibrium Inefficiency}

To compare the three cost functions, we find the optimal solution (without NE constraints) and best and worst equilibria formalizing the mathematical models presented in Section 4 with AMPL [46] and solving them with CPLEX [47]. All the results reported in this section are averaged on 100 randomly generated instances, where APs are fixed, varying the users' position.

As shown in [37], we first point out that the case for which the PoA assumes the largest values is the "corridor" topology with $m=3$ APs and $r=100$ meters. We use the name "corridor" to indicate that APs are placed along a line (the presence of walls is not considered). Numerical results show that the maximum value of PoA (over all instances) using cost function 1 is $1 . \overline{6}$ and it can be reached only with three users. Differently from PoA, the PoS is always equal to one with cost function 1, and therefore, the best equilibrium (BE) always corresponds to the optimal solution. Numerical results show that the dependency of PoA on $n$ for cost functions 2 and 3 is similar to the one of cost function 1 . The 
TABLE 3

PoS and PoA in Different Topologies

\begin{tabular}{|c|c|c|c|c|c|c|}
\hline & \multicolumn{2}{|c|}{ Uniform (a) } & \multicolumn{2}{c|}{ Non-uniform (b) } & \multicolumn{2}{c|}{ Corridor (c) } \\
\hline Function & PoS & PoA & PoS & PoA & PoS & PoA \\
\hline CF 1 & 1.00000 & 1.00733 & 1.00000 & 1.00615 & 1.00000 & 1.01156 \\
\hline CF 2 & 1.00000 & 1.00563 & 1.00020 & 1.00490 & 1.00019 & 1.00078 \\
\hline CF 3 & 1.00497 & 1.01079 & 1.00493 & 1.00696 & 1.00805 & 1.01108 \\
\hline
\end{tabular}

only significant difference concerns the maximum values of PoA at $n=3: 1.43$ for cost function 2, and 1.35 for cost function 3 (for $n>6 \mathrm{PoA}<1.1$ for all the cost functions). Differently from what happens in the case with cost function 1 , PoS with cost functions 2 and 3 can be larger than 1 and then the optimal solution may not be an equilibrium.

Moving to the analysis of realistic scenarios, in case (a) we consider a topology with $L=500 \mathrm{~m}, r=100 \mathrm{~m}, m=10$ APs, and $n=50$ users. Both users and APs are randomly deployed with a uniform distribution. The user density (measured in users $/ \mathrm{m}^{2}$ ) is $2 \cdot 10^{-4}$. In case $(\mathrm{b})$, we consider the same topology with users deployed using a nonuniform distribution. Namely, the square arena is divided into subareas and in each subarea users are deployed uniformly with different densities. In particular, we consider four quadrants with three different densities: high $\left(4 \cdot 10^{-4}\right)$, medium $\left(1.6 \cdot 10^{-4}\right)$, and low $\left(0.8 \cdot 10^{-4}\right)$. In the considered scenario, users in the bottom-left quadrant are deployed with high density, in the top-right with low density and in the other two with medium density. Finally, topology (c) is a "corridor" with $L=600, m=5$ APs, and $n=50$ uniformly distributed users (density is $1.38 \cdot 10^{-4}$ ).

Table 3 reports PoS and PoA for the three different topologies, varying the user cost function. The main result coming from this analysis is that both PoS and PoA are very close to one, regardless of the specific adopted cost function. In other words, the quality of the equilibria is very close to the optimal solution of the network selection problem. This means that in realistic settings, there is no significant difference, in terms of social cost, between the optimal solution (achievable with a centralized coordination among the users) and any NE (achievable through best response dynamics).

\subsection{Evaluating Actual Throughput and Fairness}

We provide a practical comparison of different selection strategies, evaluating the actual throughput perceived by users and the degree of fairness achievable with the proposed cost functions. For this analysis, we have fed into NS2 [7] the solutions obtained by the optimization model, representing the association of users to APs at the equilibrium. We have used NS2 version 2.33, supporting rate adaptation. The interference distance has been set to 100 meters (as APs radius) and we have changed the SNR table used for the rate adaptation, so that the associations between rate and distance shown in Table 2 are verified. Moreover, in our settings each AP works on a different frequency channel. Finally, users transmit using UDP connections at the same rate in the range [500 Kbit/s-6 Mbit/s]. Simulation results using TCP as transport protocol are reported in the supplemental material, available online [8]. Note that the case in which users are transmitting all the same rate is consistent for two

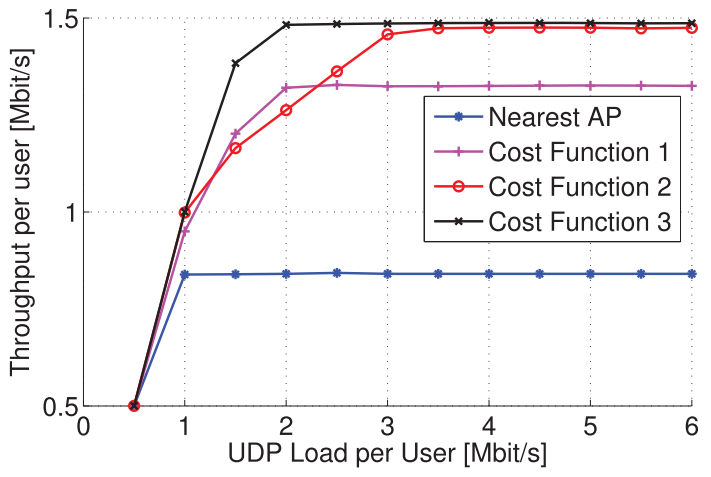

Fig. 2. Average throughput (BE) per user in 2-AP strongly asymmetric settings, increasing the UDP load.

reasons. First, because the goal of the analysis is to compare the different cost functions in terms of throughput. Second, because the considered case can be seen as the worst case scenario, i.e., for a given (maximum) rate, we derive the average throughput per user when all users are transmitting at the same (maximum) rate.

To measure the degree of fairness, we consider Jain's fairness index [48], formally:

$$
J=\frac{\left(\sum_{u \in U} \theta_{u}\right)^{2}}{n \cdot \sum_{u \in U} \theta_{u}^{2}},
$$

where $\theta_{u}$ represents the throughput of user $u$.

Moving to the details of the simulation analysis, we initially focus on realistic "strongly asymmetric settings." We consider a scenario with $m=2$ APs and $n=20$ users. All users are in the range of both APs but they are very close to one AP. We report in Fig. 2 the average throughput per user in the best equilibrium, using different cost functions and increasing the UDP load. Cost function 1 and nearest-AP lead to a lower throughput compared to the other cost functions: -10 and -45 percent, respectively. Cost functions 2 and 3 are almost equivalent in terms of throughput. We report in Fig. 3 Jain's fairness index. Cost functions 3 and nearest-AP provide the best fairness index, whereas cost function 2 leads to -23 percent and cost function 1 to -9 percent.

Hereafter, we consider the three different topologies, (a), (b), and (c), with $n=50$ users presented previously in Section 5.2. Fig. 4 reports the average throughput per user

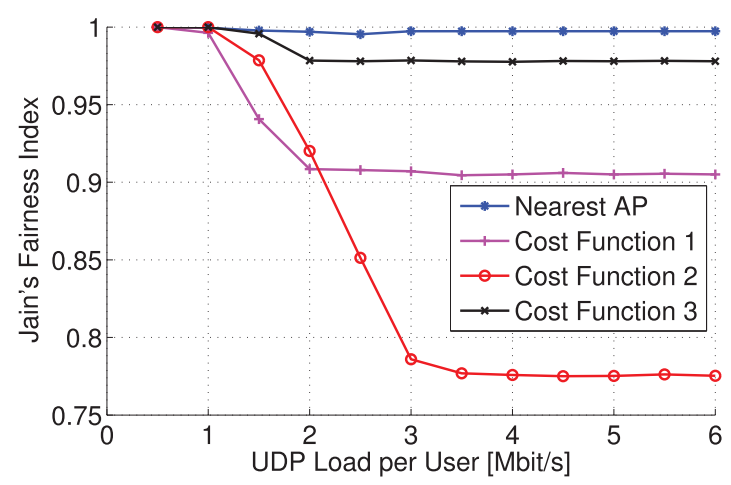

Fig. 3. Jain's fairness index (BE) in 2-AP strongly asymmetric settings, increasing the UDP load. 


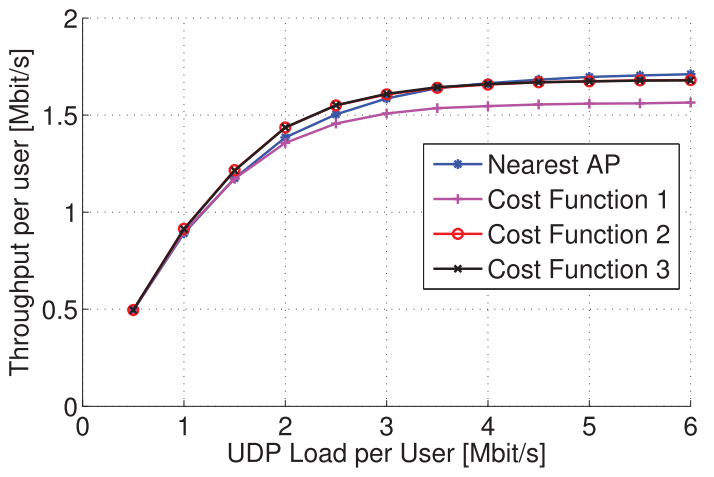

Fig. 4. Average throughput (BE) per user in uniform topologies, increasing the UDP load.

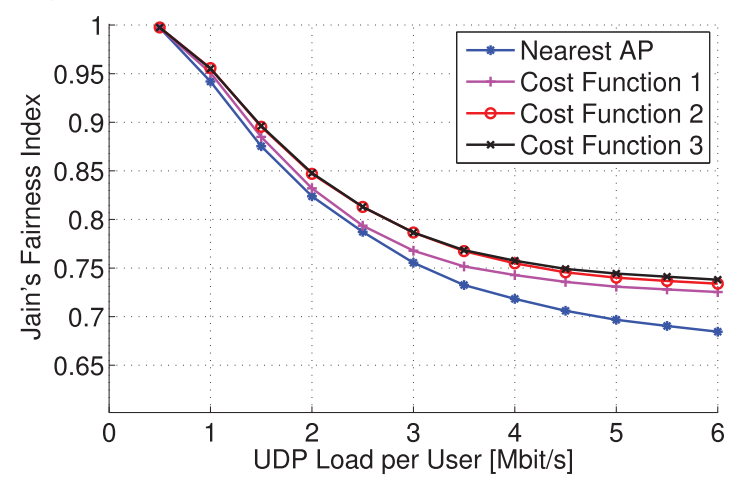

Fig. 5. Jain's fairness index (BE) in uniform topologies, increasing the UDP load.

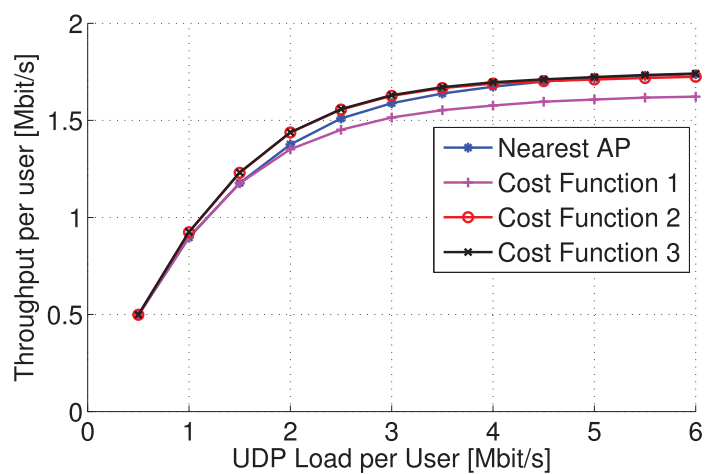

Fig. 6. Average throughput (BE) per user in nonuniform topologies, increasing the UDP load.

in the uniform scenario (a). The statistical consistency of the simulation results is evaluated in the supplemental material, available online [8]. We have observed by simulations that best equilibrium and worst equilibrium are very close in terms of actual throughput (sum over all users). This is strictly related to the fact that PoS and PoA are also close to each other (see Table 3). This confirms the goodness of the game model and, hereafter, we consider the BE only. Fig. 5 reports Jain's fairness index. We can observe that cost function 1 reaches the lowest throughput among all the function ( -8 percent w.r.t. the best case). Nearest-AP selection ensures the highest average throughput, especially increasing the UDP load, but it has the lowest degree of fairness ( -8 percent). In contrast, we can observe that cost functions 2 and 3 ensure a throughput very close to the maximum (provided by the nearest-AP selection) and, at the same time, guarantee the highest fairness.

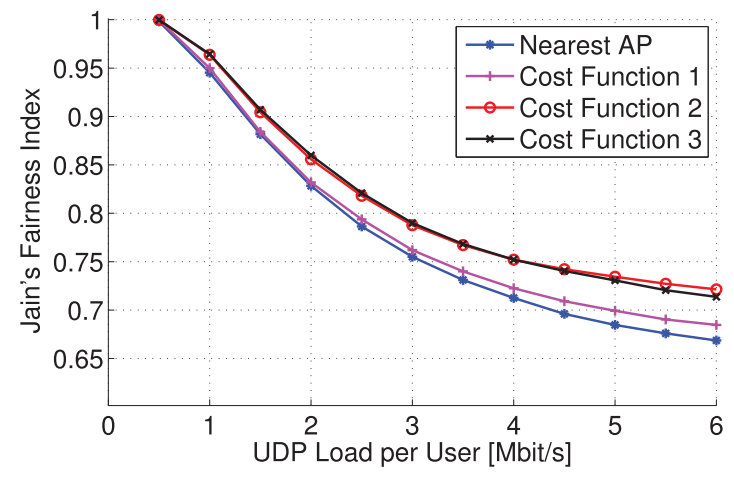

Fig. 7. Jain's fairness index (BE) in nonuniform topologies, increasing the UDP load.

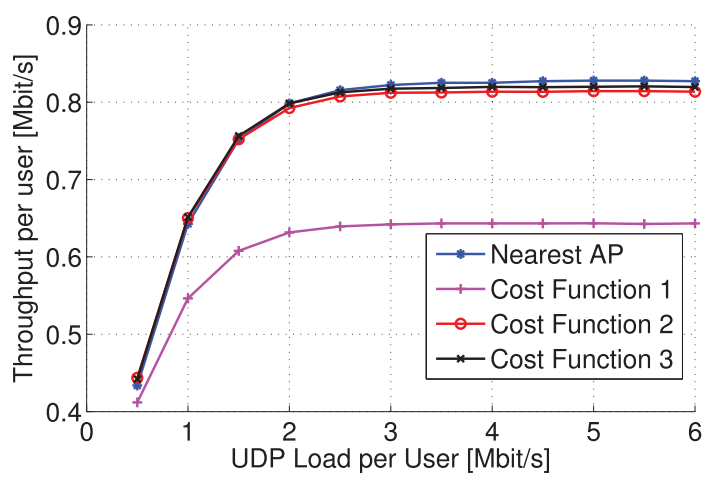

Fig. 8. Average throughput (BE) per user in corridor topologies, increasing the UDP load.

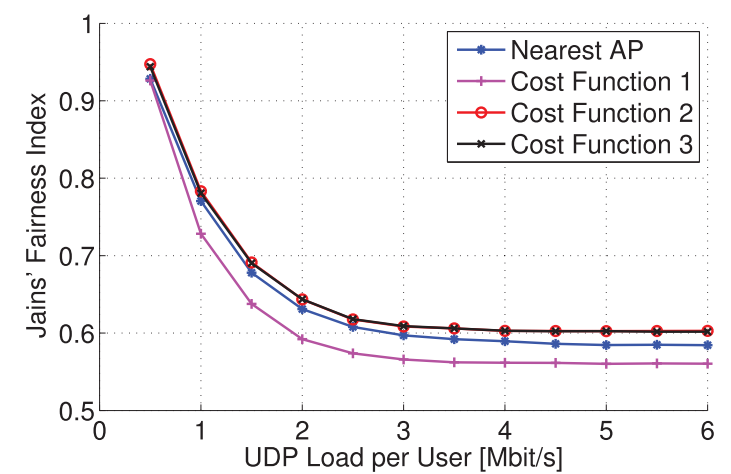

Fig. 9. Jain's fairness index (BE) in corridor topologies, increasing the UDP load.

Figs. 6 and 7 report, respectively, the average actual throughput and the Jain's fairness index under nonuniform topologies (b). Also in this case, cost functions 2 and 3 provide the highest level of fairness and the largest throughput, whereas the nearest-AP selection has the lowest fairness degree ( -6 percent from the best). The same performance curves have been obtained per user type with similar behavior (see supplemental material, available online [8]).

Finally, we consider the "corridor" topology (c) and we report in Figs. 8 and 9 the throughput and the Jain's fairness index, respectively. As in the previous cases, cost functions 2 and 3 provide the best performance. Cost function 1 leads to the lowest throughput ( -20 percent) and to the lowest fairness ( -5 percent).

Summarizing, the main outcomes of the simulation analysis are the following: 1) cost functions 2 and 3 provide 
the highest throughput and the highest fairness among all the considered functions; 2 ) nearest-AP selection leads to the lowest actual throughput in "strongly asymmetric settings" and the lowest fairness in the other tested scenarios; and 3) cost function 1 generally leads to both actual throughput and fairness lower than the ones obtained under cost function 3. The fact that cost functions 2 and 3 provide the highest throughput and fairness mean that they better reflect the quality perceived by the users, in particular with respect to common nearest-AP policy. Therefore, the implementation of these selection mechanisms in real system will lead to better performance for the users, in terms of both throughput and fairness. We finally point out that the same conclusions hold true if changing the reference propagation model, as cost functions 2 and 3 directly take into account the nominal rate assigned by the $\mathrm{AP}$, which in turn depends on the propagation model.

\subsection{Discussion on the Implementation}

It is worth analyzing the applicability of the proposed cost functions (and selection strategies) in terms of required signaling overhead. Users playing according to cost function 1 only require the information on the number of users associated with each AP, while under cost function 3 also the user's nominal rate is needed. The information of the current nominal rate can be easily available locally. Indeed, referring to IEEE 802.11 access networks, the drivers of commercial cards do provide hooks to monitor the current rate adopted for transmissions. The information on the current number of interferers should be provided by the AP itself through beaconing messages, ${ }^{3}$ or locally estimated by the users.

The calculation of cost function 2 requires each user to know also the current nominal rate adopted by all the other users associated with the same AP which would require a higher signaling overhead to be actually implemented. Therefore, even if the performance of cost functions 2 and 3 are close, cost function 2 requires the implementation of new features in real systems to provide users with the needed information. This makes cost function 3 more suitable for existing systems.

Finally, it is also worth analyzing the convergence time of the best response dynamics under the three cost functions. Such performance measure reflects the promptness of the distributed system to settle to an equilibrium. Available results for generic congestion games show that the convergence time is $O\left(n^{2} m\right)$ where $n$ is the number of users and $m$ is the number of resources [49], [50] when users cannot act simultaneously. To further assess the convergence time in our game model, we developed a best response simulator. The initial assignment of users to access points and the user that acts at each time point are chosen randomly. We generated settings as described in Section 5.2 with $n \in$ $[10,200]$ with a step of $10, m \in[3,12]$ with a step of 3 . For each parameter configuration, we generated 100 settings and we solved each setting 100 times. The convergence time (measured in number of iterations) for cost function 1 is reported in Fig. 10 (the other cost functions feature similar

3. For example, in the field of IEEE 802.11 networks, the IEEE $802.11 \mathrm{k}$ standard could be used to support the exchange of additional information.

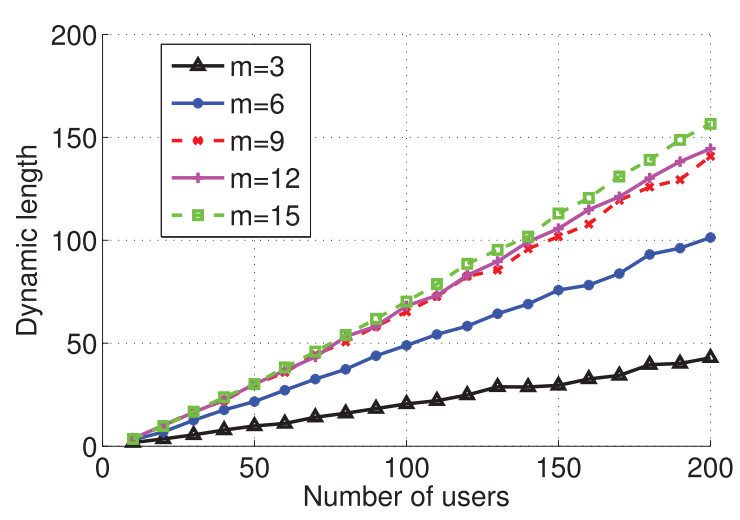

Fig. 10. Length of the dynamics with cost function 1.

behavior). The convergence time increases linearly in the number of users and it is smaller than $n$ for every $m$. Exactly, the convergence time increases in $m$ and asymptotically saturates to $\sim \frac{4}{5} n$, as $m \rightarrow+\infty$.

\section{The Network Selection Resource Allocation Game}

In the previous section, we have considered the user competition, assuming the resource allocation (i.e., frequency assignment) as fixed. Hereafter, we drop the assumption of a fixed frequency assignment, bringing the operators into the competition. In fact, in practical settings, it is reasonable to assume that each operator tries to maximize her own revenue defined, in our case, as the number of connected customers. In general, an operator may own and run several network devices (APs) to cover different-sized areas. Hereafter, we start off by considering a simplified case where a network operator only owns a single $\mathrm{AP},{ }^{4}$ which may well represent those cases where the area to be covered is limited (conference rooms, hotel lounges, etc.) and multiple APs (belonging to multiple operators) may be concurrently deployed to provide connectivity. Furthermore, for simplicity we assume that operators have complete information about the network topology. This slightly simplified but consistent scenario allows us to get insightful results on the dynamics of the competition among operators. To this end, we introduce a two-stage game model capturing also the competition among the operators. We further provide the mathematical model to solve the game, and finally discuss some numerical results, considering the different cost functions from the user side.

\subsection{Network Selection Resource Allocation Game}

The game we consider has two groups of players: users and operators. We assume that the two groups of players do not "play" simultaneously and decisions times are decoupled. Therefore, we are dealing with a (multileader/multifollower) two-stage game. First, operators (i.e., the leaders) compete selecting the most convenient frequency. Then, users (i.e., the followers) react to the APs' actions, selecting the best AP. The user game, once the frequencies have been fixed, is the same as described in previous sections. Each

4. We refer to network "operator" and "AP" indifferently. 
user tries to minimize a given cost function, $c_{u}\left(f, x_{u}^{f}\right)$, selecting the "best" operators. In contrast, APs try to maximize the number of connected users by operating at the most convenient frequency.

The equilibrium concept adopted for multistage games is the subgame perfect equilibrium, which is defined as a strategy profile which is a NE for every subgame [2].

In Section 3, we proved that a pure strategy equilibrium for the user game always exists, regardless of the specific cost function. In contrast, the existence of a pure strategy SPE for the two-stage game is guaranteed only when users adopt cost function 1. In particular, the following propositions hold true. Complete proofs are reported in the supplemental material, available online [8].

Proposition 6.1. The two-stage game with users' cost function 1 always admits a pure strategy SPE.

Proposition 6.2. The two-stage game with users' cost function 2 may not admit any pure strategy SPE.

Proposition 6.3. The two-stage game with users' cost function 3 may not admit any pure strategy SPE.

Therefore, for the two-stage game, with cost functions 2 and 3, the equilibrium is not guaranteed to always exist. However, we have shown in previous sections, that from the user side, these two functions lead to better performance, in terms of both throughput and fairness. To characterize stable states for the two-stage game even when users adopt functions 2 and 3, we introduce the concept of (pure strategy) $\epsilon$-NE and (pure strategy) $\epsilon$-SPE. An $\epsilon-\mathrm{NE}$ is a strategy profile that approximately satisfies the condition of NE. Namely, given a real nonnegative parameter $\epsilon$, a strategy profile is said to be an $\epsilon$-NE if it is not possible for any player to gain more than $\epsilon$ by unilaterally deviating from her strategy. Note that every (pure strategy) NE is an (pure strategy) $\epsilon-\mathrm{NE}$ in which $\epsilon=0$. The concept of $\epsilon$-SPE is defined similarly, allowing some player in some decision point of the game tree to play a strategy that is worse no more than $\epsilon$ with respect to the best response action. In our two-stage game, the equilibrium nonexistence problem is at the first stage game. Therefore, we allow operators to play in the first stage an $\epsilon$-NE, while we force users to play in the second stage a pure strategy NE.

\subsection{Mathematical Programming Model}

We extend the mathematical programming formulation presented in Section 4 to solve the two-stage game. The computation of an SPE requires the computation of the NEs of all the possible subgames. We denote by $\gamma$ a single subgame and by $\Gamma$ the set of all the subgames. With a slight abuse of notation, we denote by $\gamma\left(s_{a_{1}}, \ldots, s_{a_{m}}\right)$ the specific subgame induced by operators' action profile $\left(s_{a_{1}}, \ldots, s_{a_{m}}\right)$. Note that in each subgame $\gamma$ the frequency assignment is fixed, then the problem is the same as described in Sections 3 and 4 . For the case of two operators, i.e., $A=$ $\left\{a_{1}, a_{2}\right\}$ and two frequencies, i.e., $F=\left\{f_{1}, f_{2}\right\}$, it is possible to identify four different subgames. Namely, at the stage of the operators, we can have the following cases: $a_{1}$ and $a_{2}$ choose the same frequency, either $f_{1}$ or $f_{2}$, or the two operators choose different frequencies ( $a_{1}$ selects $f_{1}$ and $a_{2}$ selects $f_{2}$ or the opposite).
We can state the extended mathematical programming formulation. Parameter $b_{u a}$ remains unchanged, instead the other parameters depend also on subgame $\gamma$ as

$$
\begin{aligned}
d_{u f}^{\gamma} & = \begin{cases}1, & \text { if } u \text { can select } f \text { in } \gamma, \\
0, & \text { otherwise, }\end{cases} \\
t_{a f}^{\gamma} & = \begin{cases}1, & \text { if } a \text { transmits on } f \text { in } \gamma, \\
0, & \text { otherwise, }\end{cases} \\
i_{u u^{\prime} f}^{\gamma} & = \begin{cases}1, & \text { if } u \text { and } u^{\prime} \text { interfere on } f \text { in } \gamma, \\
0, & \text { otherwise. }\end{cases}
\end{aligned}
$$

Indeed, these parameters have the same meaning as the corresponding parameters previously introduced in Section 4, with the exception that in this case they are valid only for the specific subgame $\gamma$. With the same approach, we extend the variables on the basis of $\gamma$ as

$$
\begin{aligned}
& y_{u f}^{\gamma}= \begin{cases}1, & \text { if } u \text { chooses } f \text { in } \gamma, \\
0, & \text { otherwise, }\end{cases} \\
& s_{u a}^{\gamma}= \begin{cases}1, & \text { if } u \text { chooses } a \text { in } \gamma, \\
0, & \text { otherwise. }\end{cases}
\end{aligned}
$$

For each user $u$, we define the congestion level:

$$
\left(z_{u}^{f}\right)^{\gamma}=\sum_{v \in U} \omega_{v}^{f} y_{v f}^{\gamma} i_{u v f}^{\gamma}
$$

that is, the congestion level perceived by $u$ when selects $f$ in $\gamma$. Similarly, we define the number of users associated with each $\mathrm{AP}$, corresponding in this case to the variable that each AP $a$ wants to maximize:

$$
n_{a}^{\gamma}=\sum_{u \in U} s_{u a}^{\gamma} .
$$

Furthermore, we introduce a binary variable for every subgame $\gamma$ that assumes a value of one only when $\gamma$ is on the SPE path as

$$
h^{\gamma}= \begin{cases}1, & \text { if } \gamma \text { is on the equilibrium path } \\ 0, & \text { otherwise }\end{cases}
$$

We now describe the constraints of the model. A subset of the constraints is the same as those of the users' game described in Sections 3 and 4. In particular, the following feasibility constraints guarantee that each user selects only one network per subgame $\gamma$ :

$$
\begin{gathered}
\sum_{a \in A_{u}} s_{u a}^{\gamma}=1 \quad \forall u \in U, \gamma \in \Gamma, \\
y_{u f}^{\gamma}=\sum_{a \in A_{u}} s_{u a}^{\gamma} t_{a f}^{\gamma} \quad \forall u \in U, f \in F, \gamma \in \Gamma .
\end{gathered}
$$

And similarly, we define the NE constraints for the users, for each subgame $\gamma$ :

$$
\begin{gathered}
d_{u k}^{\gamma} y_{u f}^{\gamma} c_{u}\left(f,\left(z_{u}^{f}\right)^{\gamma}\right) \leq c_{u}\left(k,\left(z_{u}^{k}\right)^{\gamma}\right) \\
\forall u \in U, f, k \neq f \in F, \gamma \in \Gamma .
\end{gathered}
$$

We need now to define the equilibrium constraints of the operators on the basis of the users' actions in all the subgames. Since, as shown in the previous section, a twostage game may not admit any SPE in pure strategies, we 


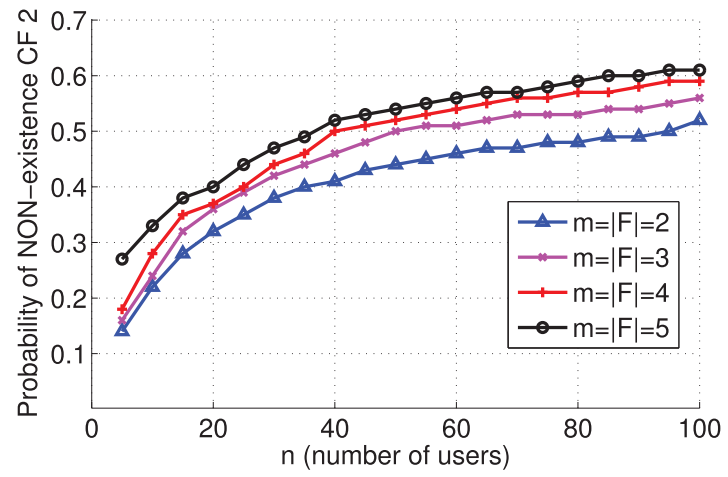

Fig. 11. Probability of nonexistence of any pure-strategy SPE when users play using cost function 2 .

need to search for the best $\epsilon$-NE (i.e., with the minimum value of $\epsilon$ ) for the first-stage game, obtaining thus an $\epsilon$-SPE for the whole game. Given a value of $\epsilon$, the $\epsilon$-Nash constraints for the operators are

$$
\begin{aligned}
n_{a}^{\gamma}+\epsilon \geq n_{a}^{\gamma^{\prime}} h^{\gamma} \quad & \forall a \in A, \gamma\left(s_{a}, s_{-a}\right) \in \Gamma, \\
& \gamma^{\prime}\left(s_{a}^{\prime}, s_{-a}\right) \in \Gamma,
\end{aligned}
$$

that is, at the (approximate) equilibrium (identified by $h^{\gamma}=1$ ) operator $a$ cannot gain more than $\epsilon$ by (unilaterally) changing her action. We need to force that there is only one subgame on the equilibrium path:

$$
\sum_{\gamma \in \Gamma} h^{\gamma}=1
$$

Finally, we want to select the best approximate equilibrium by minimizing the value of $\epsilon$ under the constraint that it is nonnegative. Therefore, we obtain

$$
\begin{aligned}
& \min \quad \epsilon \\
& \text { s.t. } \quad \epsilon \geq 0 \\
& \text { constraints }(6),(7),(8),(9),(10) .
\end{aligned}
$$

Although the above formulation has nonlinear constraints, it can be cast as a mixed-integer linear formulation, resorting to simple mathematical tricks.

\subsection{Experimental Evaluation}

We evaluate hereafter the two-stage game. In particular, we first study the existence of SPEs and the $\epsilon$ value of $\epsilon$-SPEs, then we quantify the impact of the competition among networks onto the users' perceived access costs.

\subsubsection{Existence of SPEs and Approximation Degree}

We focus on the existence of (pure strategy) SPEs with cost functions 2 and 3 and on the characterization of the best $\epsilon$-Nash approximation degree we can obtain. Cost function 1 is not considered in this analysis, since Proposition 6.1 assures the existence of SPEs. To derive SPEs and approximated SPEs, the linear model discussed in Section 6.2 is formalized in AMPL and solved using CPLEX. We generate uniform topologies, as described in Section 5.2, where the number of networks $m$ equals the number of available frequencies $|F|$ and goes from $m=|F|=2, \ldots, 5$. Furthermore, we let the number of users

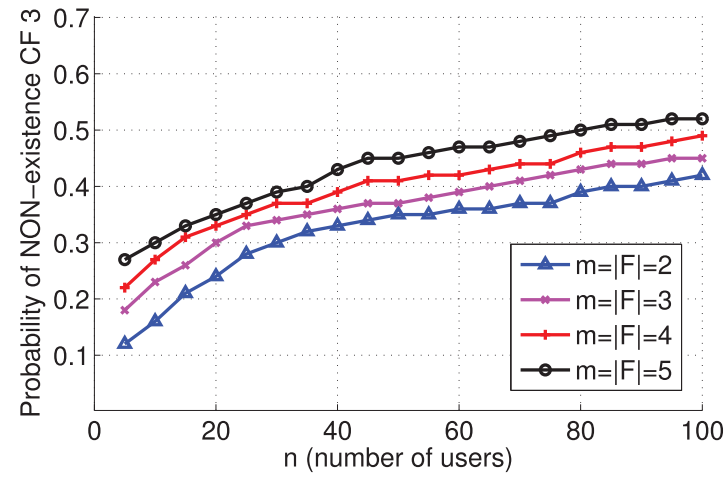

Fig. 12. Probability of nonexistence of any pure-strategy SPE when users play using cost function 3 .

go from $n=5, \ldots, 100$. All the results are averaged on 200 randomly generated instances.

First, we evaluate the average probability of nonexistence of any pure-strategy SPE, i.e., fraction of games not admitting any pure-strategy SPE. Results for cost functions 2 and 3 are reported in Figs. 11 and 12. The numerical variance can be derived by observing that the curve refers to a Binomial experiment whose variance is $p \cdot(1-p)$, where $p$ is the probability of nonexistence. The nonexistence fraction may be significative ( $>40$ percent with 100 users) under both cost functions, and generally increases with the number of users. Cost function 3 grants better results than cost function 2. As clear from the proof of Proposition 6.2, the nonexistence is due to the relative positions of APs and users. We conjecture that cost function 2 presents a space of relative positions (for which equilibrium does not exist) wider than cost function 3. Furthermore, we can observe that increasing the number of networks (and available frequencies), the probability of nonexistence increases. As done before, we conjecture that increasing the number of competitors, the strategy space for the users increases, and then also the space of relative positions increases.

Second, it is worth studying the approximation degree of $\epsilon$-SPE equilibria. To this end, we are interested in the $\epsilon$-SPEs with minimum $\epsilon$. For both cost functions, we evaluate the average minimum $\epsilon$. Figs. 13 and 14 report the average $\epsilon$ using cost functions 2 and 3, respectively. We report the statistical property of the simulation results in the supplemental material, available online [8]. The two cost functions provide very similar results. We observe that increasing the number of users, $\epsilon$ increases. This reflects the

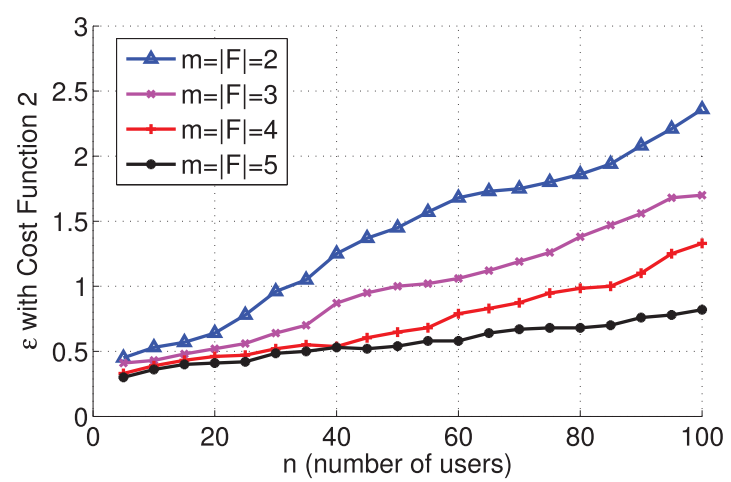

Fig. 13. Average $\epsilon$ when users play using cost function 2 . 


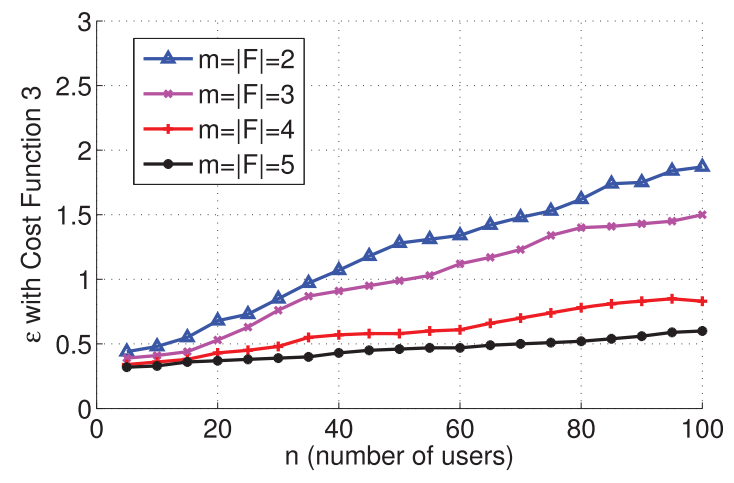

Fig. 14. Average $\epsilon$ when users play using cost function 3 .

fact that each network loses a larger number of users at the $\epsilon$-SPE as the number of users increases. However, when the number of available networks and frequencies increases, the average $\epsilon$ decreases. This highlights the fact that with a larger number of available networks, the average number of users per network decreases, and so does the average $\epsilon$. However, the loss of users for each network at the $\epsilon$-SPE is always small compared to $n$ ( $\leq 3$ percent). Note that for the sake of completeness, we have also run simulations on cases in which the number of available frequencies is less than the number of operators. Namely, given $m$, we have evaluated all the cases for $|F|=2, \ldots, m$ (see supplemental material, available online [8]). We observed that the average $\epsilon$ decreases when the number of available frequencies reduces. This can be explained by the fact that in this case the space of relative positions decreases.

\subsubsection{Quality of the $\epsilon$-SPES for the Users}

We evaluate the efficiency of (approximate) equilibria in terms of users' social costs to understand how the competition between operators affects the quality perceived by the users. In our analysis, we do not consider the operators' utility, because every equilibrium is Pareto efficient for the operators (their utilities sum up to the total number of users).

Under the same settings as in the previous section, we find the optimal (approximate) equilibrium for the users among those that minimize $\epsilon$. In addition, we find the equilibria of the users' game when operators are forced to play different frequencies (corresponding to the case in which the users' cost is minimum) and the same frequency (corresponding to the case in which the users' cost is maximum), respectively.

Fig. 15 shows the average cost per user calculated according to cost functions 2 and 3 for the equilibria of games played according to cost functions 2 and 3, when the number of operators and the number of frequencies are both equal to 2 . Two different scales are used for the values of the two different cost functions. The results have been scaled such that the lines corresponding to the situations in which operators use the same frequency with cost functions 2 and 3 overlap and the same when operators use different frequencies. Increasing the number of networks / frequencies, we obtained similar results.

The main result is that the competition among operators affects the cost of the users. In fact, even if the rate is close to the case where different frequencies are used, the number of

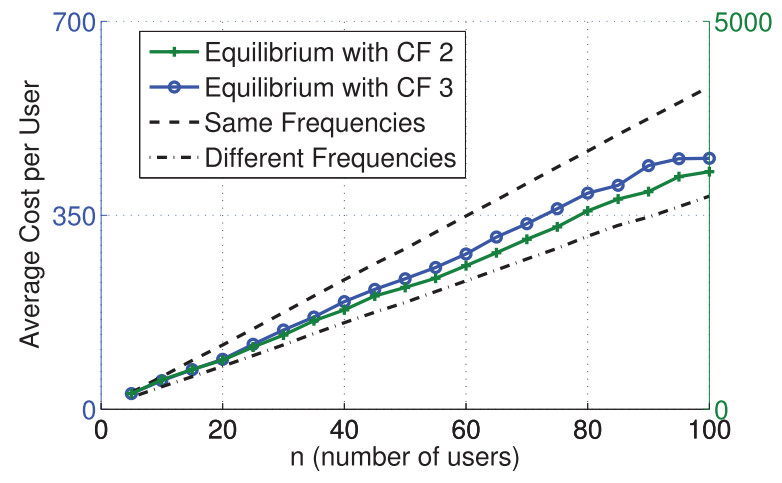

Fig. 15. Average cost per user when $m=|F|=2$.

interferers increases. This leads to an increase in the usersperceived cost for both cost functions 2 and 3. This result is confirmed by the evaluation of the actual throughput (by NS2 based simulations) that shows a behavior similar to the curves in Fig. 15.

\section{Concluding Remarks}

Motivated by the heterogeneity of modern wireless access networks, we proposed in this paper a study to capture the dynamics among end users and network operators in the processes of network selection and resource allocation. We resorted to noncooperative game theory to model the competition among multiple end users in accessing shared wireless networks. We analyzed three game models that differ in the access cost functions, each end user tends to minimize and reasonably represent three realistic cases of wireless access technologies. To solve the games, we proposed a mathematical programming formulation for the network selection problem, which is able to return the best and worst equilibria for a thorough evaluation of the equilibria quality in terms of Price of Anarchy and Price of Stability.

We have then included the operators in the network selection process by letting each operator dynamically plan its radio resources. Namely, we proposed a two-stage game model where operators play first by competitively setting their operating frequencies to capture the highest number of end users, which, in turn, play the aforementioned network selection games in the second stage of the game. Formal results on the existence of subgame perfect equilibria have been derived for all the game instances. Even if the proposed two-stage game may not always admit any pure strategy equilibrium, it is possible to enforce approximated equilibria with a reasonably small quality loss. Thus, the proposed game model can be suitably adopted to represent and drive the dynamics of practical network scenarios.

The main results of the analysis are as follows:

- best response dynamics in the network selection based on the perceived interference and nominal rate tend to converge to socially optimal solution;

- the equilibria obtained playing with a multiparameter cost function (perceived interference and nominal rate) feature better performance (actual throughput and fairness) than if playing with single parameter cost functions (either rate or interference) especially under asymmetric network scenarios; 
- cost function 3 is the best tradeoff between performance and implementability to develop protocols for users' network selection;

- competition among operators may be dangerous for two reasons: first, the two-stage game may not admit any (pure-strategy) equilibrium, second, it leads, in general, to worse performance for the users.

Natural follow up of this work includes the extension to the case in which the network selection process accounts also for information on the type of traffic required by the users, each operator owns multiple access points and/ or has statistical information on the users' distribution, the impact of connection fees onto the game theoretic model and the implementation of network selection/ association and resource allocation protocols to enforce best response dynamics.

\section{REFERENCES}

[1] E. Gustafsson and A. Jonsson, "Always Best Connected," IEEE Wireless Comm., vol. 10, no. 1, pp. 49-55, Feb. 2003.

[2] D. Fudenberg and J. Tirole, Game Theory. MIT, 1991.

[3] R.W. Rosenthal, "A Class of Games Possessing Pure-Strategy Nash Equilibria," Int'l J. Game Theory, vol. 2, no. 1, pp. 65-67, 1973.

[4] N. Nisan, T. Roughgarden, E. Tardos, and V.V. Vazirani, Algorithmic Game Theory. Cambridge Univ., 2007.

[5] E. Anshelevich, A. Dasgupta, J. Kleinberg, E. Tardos, T. Wexler, and T. Roughgarden, "The Price of Stability for Network Design with Fair Cost Allocation," Proc. IEEE 45th Ann. Symp. Foundations Computer Science (FOCS), pp. 59-73, 2004.

[6] E. Koutsoupias and C. Papadimitriou, "Worst-Case Equilibria," Proc. 16th Ann. Conf. Theoretical Aspects Computer Science (STACS), pp. 404-413, 1999.

[7] "The Network Simulator - ns-2," http:/ /www.isi.edu/nsnam/ns, 2013.

[8] M. Cesana, N. Gatti, and I. Malanchini, "Network Selection and Resource Allocation Games for Wireless Access Networks," IEEE Trans. Mobile Computing, supplemental material, doi:10.1109/ TMC.2012.207, Nov. 2012.

[9] O. Ormond, J. Murphy, and G. Muntean, "Utility-Based Intelligent Network Selection in Beyond 3G Systems," Proc. IEEE Int'l Conf. Comm. (ICC), pp. 1831-1836, 2006.

[10] K. Premkumar and A. Kumar, "Optimum Association of Mobile Wireless Devices with a WLAN-3G Access Network," Proc. IEEE Int'l Conf. Comm. (ICC), pp. 2002-2008, 2006.

[11] W. Shen and Q.-A. Zeng, "Cost-Function-Based Network Selection Strategy in Integrated Wireless and Mobile Networks," IEEE Trans. Vehicular Technology, vol. 57, no. 6, pp. 3778-3788, Nov. 2008

[12] Q. Song and A. Jamalipour, "Network Selection in an Integrated Wireless LAN and UMTS Environment Using Mathematical Modelling and Computing Techniques," IEEE Wireless Comm. vol. 12, no. 3, pp. 42-48, June 2005.

[13] D. Charilas, O. Markaki, D. Nikitopoulos, and M. Theologou, "Packet-Switched Network Selection with the Highest QoS in 4G Networks," Computer Networks, vol. 52, no. 1, pp. 248-258, 2008.

[14] F. Bari and V. Leung, "Automated Network Selection in a Heterogeneous Wireless Network Environment," IEEE Network, vol. 21, no. 1, pp. 34-40, Jan./Feb. 2007.

[15] M. Bernaschi, F. Cacace, G. Iannello, S. Za, and A. Pescape, "Seamless Internetworking of WLANs and Cellular Networks: Architecture and Performance Issues in a Mobile IPv6 Scenario," IEEE Wireless Comm., vol. 12, no. 3, pp. 73-80, June 2005.

[16] Y. Bejerano, S.-J. Han, and L. Li, "Fairness and Load Balancing in Wireless LANs Using Association Control," IEEE ACM Trans. Network, vol. 15, no. 3, pp. 560-573, June 2007.

[17] N. Blefari-Melazzi, D.D. Sorte, M. Femminella, and G. Reali, "Autonomic Control and Personalization of a Wireless Access Network," Computer Network, vol. 51, no. 10, pp. 2645-2676, 2007.

[18] Y. Lee and S.C. Miller, "Network Selection and Discovery of Service Information in Public WLAN Hotspots," Proc. ACM Int'l Workshop Wireless Mobile Applications and Services WLAN Hotspots (WMASH), pp. 81-92, 2004.
[19] Y. Fukuda and Y. Oie, "Decentralized Access Point Selection Architecture for Wireless LANs," IEICE Trans. Comm., vol. E90-B, no. 9, pp. 2513-2523, Sept. 2007.

[20] H. Gong, K. Nahm, and J. Kim, "Distributed Fair Access Point Selection for Multi-Rate IEEE 802.11 WLANs," IEICE Trans. Information and Systems, vol. E91-D, no. 4, pp. 1193-1196, Apr. 2008

[21] S. Vasudevan, K. Papagiannaki, C. Diot, J. Kurose, and D. Towsley, "Facilitating Access Point Selection in IEEE 802.11 Wireless Networks," Proc. ACM Fifth SIGCOMM Conf. Internet Measurement (IMC), pp. 293-298, 2005.

[22] J. Antoniou, V. Papadopoulou, and A. Pitsillides, "A Game Theoretic Approach for Network Selection," technical report, 2008.

[23] R. Trestian, O. Ormond, and G.-M. Muntean, "Game Theory Based Network Selection: Solutions and Challenges," IEEE Comm. Surveys and Tutorials, vol. 14, no. 4, pp. 1212-1231, Fourth Quarter 2012.

[24] K. Mittal, E.M. Belding, and S. Suri, "A Game-Theoretic Analysis of Wireless Access Point Selection by Mobile Users," Computer Networks, vol. 31, no. 10, pp. 2049-2062, 2008.

[25] L.-H. Yen, J.-J. Li, and C.-M. Lin, "Stability and Fairness of AP Selection Games in IEEE 802.11 Access Networks," IEEE Trans. Vehicular Technology, vol. 60, no. 3, pp. 1150-1160, Mar. 2011.

[26] F. Xu, C. Tan, Q. Li, G. Yan, and J. Wu, "Designing a Practical Access Point Association Protocol," Proc. IEEE INFOCOM, pp. 1-9, 2010.

[27] L. Chen, "A Distributed Access Point Selection Algorithm Based on No-Regret Learning for Wireless Access Networks," Proc. IEEE 71st Vehicular Technology (VTC), pp. 1-5, 2010.

[28] V. Gajic, H. Jianwei, and B. Rimoldi, "Competition of Wireless Providers for Atomic Users: Equilibrium and Social Optimality," Proc. 47th Ann. Allerton Conf. Comm., Control, and Computing (Allerton '09), pp. 1203-1210, 2009.

[29] S. Shakkottai, E. Altman, and A. Kumar, "Multihoming of Users to Access Points in WLANs: A Population Game Perspective," IEEE J. Selected Areas in Comm., vol. 25, no. 6, pp. 1207-1215, Aug. 2007

[30] L. Jiang, S. Parekh, and J. Walrand, "Base Station Association Game in Multi-Cell Wireless Networks (Special Paper)," Proc. IEEE Wireless Comm. Networking Conf. (WCNC), pp. 1616-1621, 2008.

[31] N. Kaci, P. Maille, and J.-M. Bonnin, "Performance of Wireless Heterogeneous Networks with Always-Best-Connected Users," Proc. Euro-NGI Conf. Next Generation Internet (NGI) Networks, pp. 18, 2009.

[32] D. Niyato and E. Hossain, "A Noncooperative Game-Theoretic Framework for Radio Resource Management in 4G Heterogeneous Wireless Access Networks," IEEE Trans. Mobile Computing, vol. 7, no. 3, pp. 332-345, Mar. 2008.

[33] M. Khan, A. Toker, C. Troung, F. Sivrikaya, and S. Albayrak, "Cooperative Game Theoretic Approach to Integrated Bandwidth Sharing and Allocation," Proc. Int'l Conf. Game Theory Networks (GameNet), pp. 1-9, May 2009.

[34] D. Niyato and E. Hossain, "QoS-Aware Bandwidth Allocation and Admission Control in IEEE 802.16 Broadband Wireless Access Networks: A Non-Cooperative Game Theoretic Approach," Computer Networks, vol. 51, no. 7, pp. 3305-3321, Aug. 2007.

[35] J. Antoniou and A. Pitsillides, "4G Converged Environment: Modeling Network Selection as a Game," Proc. 16th ICT Mobile Wireless Comm. Summit, pp. 1-5, 2007.

[36] L. Berlemann, G. Hiertz, B. Walke, and S. Mangold, "Radio Resource Sharing Games: Enabling QoS Support in Unlicensed Bands," IEEE Network, vol. 19, no. 4, pp. 59-65, July/Aug. 2005.

[37] M. Cesana, N. Gatti, and I. Malanchini, "Game Theoretic Analysis of Wireless Access Network Selection: Models, Inefficiency Bounds, and Algorithms," Proc. Workshop Game Theory and Comm. Networks (GAMECOMM), 2008.

[38] M. Cesana, I. Malanchini, and A. Capone, "Modelling Network Selection and Resource Allocation in Wireless Access Networks with Non-Cooperative Games," Proc. IEEE Fifth Int'l Conf. Mobile Ad Hoc and Sensor Systems (MASS), pp. 404-409, 2008.

[39] I. Milchtaich, "Congestion Games with Player-Specfic Payoff Functions," Games Economic Behavior, vol. 13, no. 1, pp. 111-124, 1996.

[40] I. Malanchini, M. Cesana, and N. Gatti, “On Spectrum Selection Games in Cognitive Radio Networks," Proc. IEEE Global Comm Conf. (GlobeCom), pp. 1-7, 2009. 
[41] G. Judd, X. Wang, and P. Steenkiste, "Efficient Channel-Aware Rate Adaptation in Dynamic Environments," Proc. Sixth ACM Int'l Conf. Mobile Systems, Applications, and Services (MobiSys '08), pp. 118-131, http://doi.acm.org/10.1145/1378600.1378615, 2008.

[42] K. Gilhousen, I. Jacobs, R. Padovani, A.J. Viterbi, L.A. Weaver, and C.E. Wheatley, "On the Capacity of a Cellular CDMA System," IEEE Trans. Vehicular Technology, vol. 40, no. 2, pp. 303-312, May 1991.

[43] M. Heusse, F. Rousseau, G. Berger-Sabbatel, and A. Duda, "Performance Anomaly of 802.11b," Proc. IEEE INFOCOM, vol. 2, pp. 836-843, 2003.

[44] I. Milchtaich, "Weighted Congestion Games with Separable Preferences," Games Economic Behavior, vol. 67, no. 2, pp. 750757, 2009.

[45] D.M. Pozar, Microwave Engineering, fourth ed. John Wiley \& Sons, 2004.

[46] R. Fourer, D. Gay, and B. Kernighan, AMPL: A Modeling Language for Mathematical Programming. Thomson/Brooks/Cole, 1993.

[47] ILOG CPLEX 10.0 User's Manual, http://www.ilog.com/ products/cplex, 2013.

[48] R. Jain, D. Chiu, and W. Hawe, "A Quantitative Measure of Fairness and Discrimination for Resource Allocation in Shared Computer Systems," Technical Report TR-301, DEC Research Report, 1984

[49] S. Ieong, R. McGrew, E. Nudelman, Y. Shoham, and Q. Sun, "Fast and Compact: A Simple Class of Congestion Games," Proc. 20th Nat'l Conf. Artificial Intelligence (AAAI), 2005.

[50] H. Ackermann, H. Roglin, and B. Vocking, "On the Impact of Combinatorial Structure on Congestion Games," J. ACM, vol. 22, no. 6, article 25, 2008.

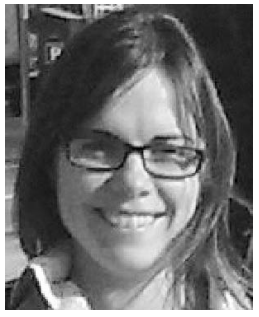

Ilaria Malanchini received the BS and MS degrees in telecommunications engineering from Politecnico di Milano, Italy, in 2005 and 2007, respectively. In December 2011, she received the $\mathrm{PhD}$ degree in electrical engineering from both Drexel University and Politecnico di Milano. Currently, she is a postdoctoral researcher at Politecnico di Milano. Her research interests focus on optimization models, mathematical programming, game theory, and stochastic geometry, with the application of these techniques to wireless network problems. She is a member of the IEEE and the IEEE Computer Society.

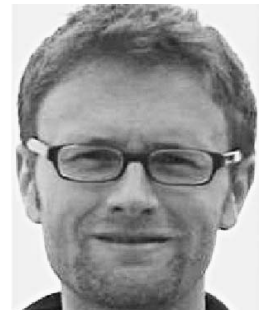

Matteo Cesana received the MS degree in telecommunications engineering and the $\mathrm{PhD}$ degree in information engineering from the Politecnico di Milano in 2000 and 2004 respectively. From 2002 to 2003, he was a visiting researcher with the Computer Science Department at the University of California, Los Angeles. He is currently an assistant professor with the Dipartimento di Elettronica e Informazione, Politecnico di Milano. His research activities are in the field of performance evaluation of cellular systems, ad hoc networks protocol design and evaluation, and wireless networks optimization. He is a member of the IEEE and the IEEE Computer Society.

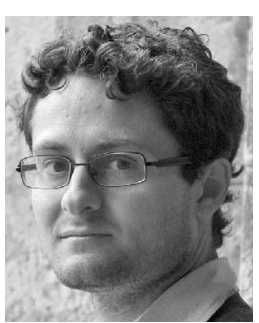

Nicola Gatti received the MS degree in biomedical engineering and the PhD degree in information engineering from Politecnico di Milano, Italy, in 2001 and 2005, respectively. Since 2006, he has been an assistant professor in computer science at the Politecnico di Milano. His research interests include algorithmic game theory, computational microeconomics, artificial intelligence, and operations research. In 2011 he received the AIxIA award as a best young Italian researcher in artificial intelligence.

$\triangleright$ For more information on this or any other computing topic, please visit our Digital Library at www.computer.org/publications/dlib. 